\title{
Análisis de la distribución de contenidos electrónicos de Wiley InterScience
}

\author{
Por Ma Luisa Alvite Díez y Blanca Rodríguez Bravo
}

\begin{abstract}
Resumen: Análisis cualitativo de Wiley InterScience, servicio de la editorial Wiley \& Sons dedicado a la provisión de contenidos científicos electrónicos, referenciales y a texto completo, contratado mayoritariamente por las bibliotecas universitarias españolas con el fin de evaluar su cobertura informativa, arquitectura de acceso, prestaciones de búsqueda, características de la interfaz, funcionalidades y servicios de valor añadido a partir de la suscripción existente en Bucle, consorcio de bibliotecas universitarias de Castilla y León.
\end{abstract}

Palabras clave: Evaluación, Interfaces, Proveedores de contenidos electrónicos, Publicaciones electrónicas, Wiley InterScience.

Title: Analysis of Wiley InterScience, provider of electronic contents

Abstract: A qualitative analysis of Wiley InterScience, service of Wiley \& Sons publisher providing electronic scientific contents, references and full texts, subscribed to mainly by Spanish academic libraries. The purpose of this analysis is to evaluate the information coverage, access architecture, search facilities, interface characteristics, functionalities and added value services, based on the subscription held by Bucle, the consortium of Castilla y León academic libraries.

Keywords: Evaluation, Interfaces, Electronic content providers, Electronic publications, Wiley InterScience.

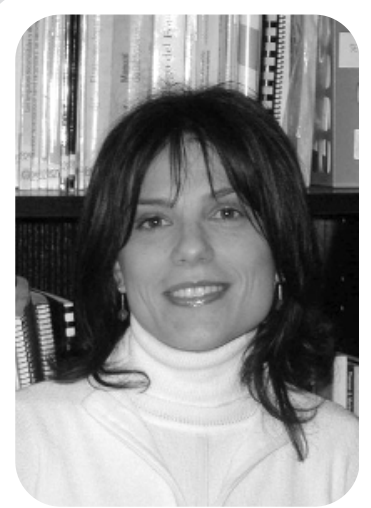

Ma Luisa Alvite Díez es diplomada en biblioteconomía y documentación, licenciada en filosofía y letras (sección filología) y doctora en filología hispánica por la Universidad de León. Profesora asociada del Área de Biblioteconomía y Documentación de la Universidad de León. Sus líneas actuales de investigación se hallan relacionadas con la recuperación de información, la documentación jurídica y el estudio de interfaces de usuario. Ha publicado varios trabajos relacionados con estas líneas en diferentes congresos y revistas.

Blanca Rodríguez Bravo es licenciada en geografía e historia por la Universidad A utónoma de Madrid, doctora en historia por la Universidad de León, postgrado en documentación por la Universidad Complutense y en documentación digital por la Universidad Pompeu Fabra. Profesora titular de universidad del Área de Biblioteconomía y Documentación de la Universidad de León. Sus líneas actuales de investigación se hallan relacionadas con la representación de información, la organización del conocimiento y el estudio de interfaces de usuario. Ha publicado dos libros y varios

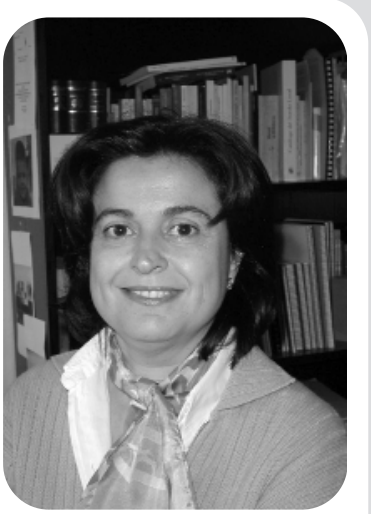
trabajos relacionados con estas líneas en diferentes congresos y revistas.

\section{Introducción}

ESTE TRABAJO SE ENMARCA dentro de un proyecto de investigación subvencionado por la Universidad de León ${ }^{1}$ cuyo objetivo general es la evaluación de los contenidos digitales proporcionados por las bibliotecas universitarias españolas en orden a lograr un conocimiento riguroso de su accesibilidad, modalidades de gestión y uso de los mismos por parte de la comuni- dad investigadora. El análisis se realiza partiendo de las suscripciones mantenidas por el consorcio de Castilla y León (Bucle).

El proyecto nos proporcionará un conocimiento global de la pro- 
visión de información electrónica, su distribución y uso por parte de la comunidad científica, lo que redundará en beneficio del fortalecimiento de la negociación de las bibliotecas universitarias con los proveedores de recursos electrónicos y en la toma fundamentada de decisiones en la contratación e inversión de presupuestos limitados.

La finalidad última de las bibliotecas universitarias no es otra que la provisión de información. El entorno electrónico en el que se hallan inmersas ha modificado en gran medida este papel trascendental en la política de investigación y desarrollo de un país, creemos que relegando algunas de las funciones bibliotecarias a favor de empresas con claros criterios comerciales, auténticos monopolios editoriales que comercializan grandes colecciones temáticas.

\section{«WIS facilita el acce- so a los contenidos mediante un formula- rio de búsqueda y a través de la navega- ción por 14 categorí- as temáticas y por ti- pos de fuentes de in- formación»}

La primera solución adoptada ante esta situación ha sido la creación de consorcios de bibliotecas que fortalezcan la negociación con los intermediarios (distribuidores de contenidos) tratando de rentabilizar los menguados presupuestos, optimizar recursos y adquisiciones. Los agentes comerciales lanzan nuevos modelos de comercialización a través de licencias ajustadas a la complejidad técnica y a las necesidades de información de todos los miembros del consorcio y negociadas individualmente por cada uno de ellos según las características y el volumen de sus componentes.
El presente estudio analiza de modo aislado un proveedor de contenidos electrónicos y se concibe, por tanto, como un primer paso dentro de un proyecto más ambicioso. Se trata de evaluar el servicio Wiley InterScience (WIS) de John Wiley \& Sons, tradicional empresa editora, fundada en 1807 , especializada en la publicación de libros y revistas de ciencia, tecnología y medicina, que además de ofertar acceso electrónico referencial a través de bases de datos, comercializa sumarios y textos completos de revistas y, asimismo, su fondo editorial de libros y obras de referencia en formato digital. $\mathrm{Bu}$ cle tiene contratado el paquete de contenidos electrónicos a través de Ebsco.

WIS resulta ejemplar en la ilustración de las tres plataformas mayoritarias de acceso a los contenidos electrónicos científico-técnicos: revistas electrónicas, bases de datos referenciales y e-books.

\section{Objetivos y metodología}

Los fines concretos del presente trabajo son los siguientes:

-Evaluar la cobertura informativa de WIS.

-Examinar las diversas prestaciones de búsqueda.

- Analizar la interfaz de usuario y valores añadidos de los distintos servicios.

La exploración del paquete electrónico de WIS se ha llevado a cabo durante la segunda quincena del mes de enero y primera de febrero de 2004. Con el fin de aplicar técnicas y métodos que permitan obtener información fiable para la toma de decisiones, hemos articulado el proceso evaluativo en una serie de parámetros e indicadores. No obstante, no hemos asignado valores dado que para acometer una evaluación cuantitativa resultaría más adecuado un análisis comparativo entre diversos suministradores de contenidos.

\section{Extensión de los conteni-} dos.

-Cobertura horizontal: volumen de publicaciones electrónicas y bases de datos.

-Cobertura temática: disciplinas científicas recogidas.

-Cobertura vertical: alcance retrospectivo de los contenidos.

Para la evaluación de los contenidos se ha utilizado tanto el acceso navegacional temático como los accesos por tipo de productos informativos: revistas, libros, obras de referencia, manuales de laboratorio y bases de datos, accediendo de modo individualizado a cada título de revista o libro para extraer los datos que figuran en el apartado de cobertura vertical.

\section{Arquitectura de acceso.}

- Sistemas de control de acceso empleado: password, autenticación de direcciones IP, certificados digitales, PAPI, superusuarios, etc.

-Prestaciones de búsqueda y de navegación: niveles de búsqueda, categorías navegacionales, campos de búsqueda, búsqueda por palabra clave, análisis del lenguaje de recuperación, operadores, reutilización de estrategias de búsqueda, etc.

—Presentación de resultados: datos descriptivos, criterios para la ordenación de los resultados y posibilidades de ordenación de los mismos, formatos de las publicaciones, etc.

Se han realizado diversas consultas para conocer la potencia de las herramientas de recuperación, los formularios de búsqueda sencilla y avanzada, la profundidad de la búsqueda exploratoria, las relaciones entre productos distribuidos o las herramientas específicas de algunos de los contenidos. Nos hemos registrado como usuarios del 
net Library Lider en libros electrónicos

Como principol proveedor de fibros electrónicos, netlibrary ayuda a Universidades, bibliotecas públicas y centros especializados a crear un entorno de aprendizaje mejor y más eficiente.

Descubrir
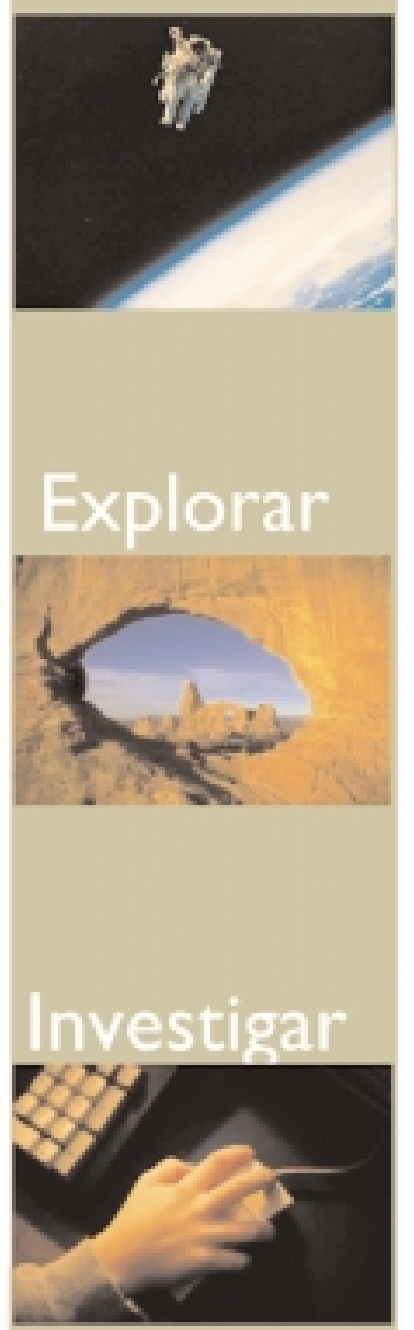

El catálogo de netLibrary contiene más de 60,000 títulos de los editores más importantes de todo el mundo.

\section{Acceso a miles de eBooks a través de una única y fácil} Interfaz, en español

\section{Desde cualquier lugar, a cualquier hora}

Potente herramienta de búsqueda facilita la búsqueda a través de todos los títulos de que dispone

Préstamo automático. No hay pérdidas, ni robos o libros sin devolver o que se deterioran

Métodos de formación actuales - incluyendo educación a distancia

Contenido fiable reconocido por su calidad

\section{Visitenos en: www.netlibrary.com}

Catálogo online: www.netlibrary.com/titleselect
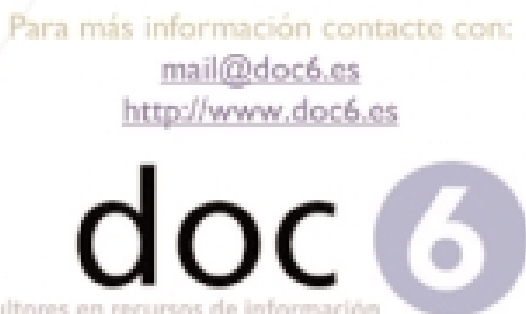

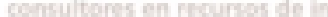

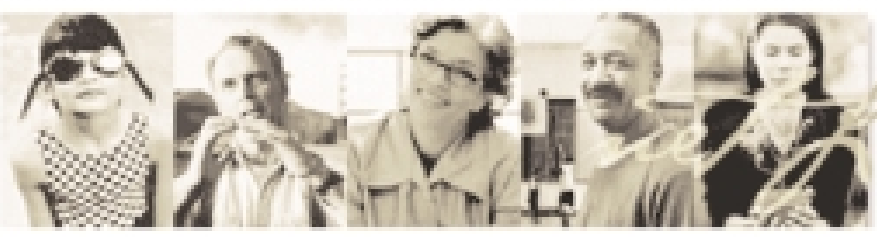

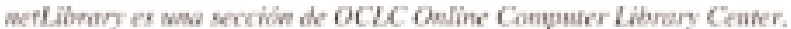

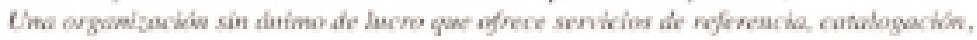

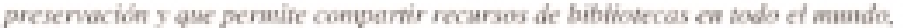


sistema para tener acceso a posibilidades sólo previstas en estas circunstancias.

\section{Características de la inter-} faz.

-Diseño: instrucciones de uso sencillas, correcta visualización, disposición adecuada de la información, resalte de elementos, etc.

-Ergonomía: características y posibilidades de selección, rapidez y calidad de descarga e impresión de registros, posibilidades de personalización de la página de acceso.

-Amigabilidad: sintaxis de los mensajes, mensajes de error, carácter y adecuación de ayudas, posibles sugerencias del sistema.

Se han empleado las búsquedas realizadas para valorar las características de la interfaz, su adecuación al usuario, en particular al registrado en WIS, se han provocado mensajes de error y repasado los sistemas de ayuda.

\section{Funcionalidades y servi- cios de valor añadido.}

-Alerta: recepción periódica vía correo electrónico de información sobre las publicaciones que atienden al perfil de usuario establecido.

-Difusión selectiva de la información: envío regular de novedades a cuentas personales atendiendo a búsquedas avanzadas del usuario.

-Pre-impresión: acceso en línea con anterioridad a la edición de la revista.

—Estadísticas de uso: de notable importancia para las bibliotecas que contratan estos servicios.

—Dosieres de cursos: paquetes pedagógicos de recursos electrónicos seleccionados.

-Técnicas de enlazado empleadas.
—Funcionalidades bibliométricas.

Hemos creado un perfil de usuario para la solicitud de alertas y DSI; en cuanto al resto de los puntos nos hemos basado en las características que ofrece la licencia contratada y la información que la empresa presenta en el apartado de "Servicios al bibliotecario".

\section{Extensión de los contenidos}

WIS destaca, frente a otros proveedores de contenidos electrónicos, por ofrecer un importante número de libros electrónicos a sus suscriptores. Mayoritariamente títulos de su propio fondo editorial y un escaso número de coediciones.

Resulta difícil delimitar los contenidos ofertados a partir de los accesos navegacionales que se proponen al usuario, ya que únicamente se accede a un listado completo con datos descriptivos de las revistas, libros electrónicos, obras de referencia y bases de datos desde el apartado dedicado a los servicios para bibliotecarios. La información aportada en esta ubicación resulta, sin embargo, muy completa en el caso de las revistas pues se recogen datos identificativos, nuevos contenidos y cambios en las publicaciones junto con facilidades de acceso y precio.

Asimismo, es problemático conocer la oferta real de contenidos distribuidos accesibles para licenciatarios de la EAL (Enhanced Access License, licencia de acceso mejorado) que es la ideada para los consorcios y que supera la cobertura de la BAL (Basic Access License, de acceso básico). Parecería lógico que la licencia suscrita especificara los contenidos accesibles a texto completo y aquéllos de los que sólo se consiguen resúmenes o tablas de contenido gratuitamente. Conviene aclarar que muchos de los contenidos ofertados sólo permiten su acceso completo median- te el uso de bonos (token) por medio del servicio Article Select, o pagando con tarjeta de crédito payper-view. Es el caso mayoritario de los libros electrónicos (de los que sólo permite descargar libremente la tabla de contenidos, la introducción y los resúmenes de los capítulos) y de los manuales de laboratorio. Lo mismo acontece ocasionalmente en los demás productos.

WIS facilita el acceso a los contenidos mediante un formulario de búsqueda y a través de la navegación por 14 categorías temáticas y por tipos de fuentes de información: Journals, Online books, Reference works, Databases, Current protocols y Collections. En el apartado de colecciones ofrece opciones de suscripción a paquetes temáticos específicos de contenidos ofrecidos en los bloques anteriores.

Parece que la intención es dirigir al usuario a la navegación por categorías temáticas preferentemente que es la que se muestra de modo destacado en pantalla, precisando el uso del scroll para el acceso diferenciado por materiales.

Asimismo, es preciso reseñar que la cobertura lingüística de los contenidos se circunscribe al inglés y al alemán, aunque éste en menor medida. Destacamos positivamente la profundidad en la digitalización que no se limita a los artículos científicos, ofreciendo editoriales, reseñas, etc.

Para el análisis del contenido, como se ha señalado, hemos decidido estudiar tres subparámetros: la cobertura horizontal, temática y vertical.

\section{Cobertura horizontal.}

Pretendemos en este parámetro recoger el número de ítems ofrecidos por Wiley distinguiendo los bloques preferentes que la propia editorial establece. Además, hemos optado por diferenciar en el apartado de revistas electrónicas, 
las abiertas de las cerradas o cerradas con backfile que WIS agrupa en un único bloque.

Los datos que se presentan, accediendo singularmente a cada uno de los contenidos, no coinciden exactamente con los ofrecidos por el distribuidor ni desde el apartado de "Servicios para bibliotecarios" ni desde los accesos navegacionales, ya sea el temático o el de tipo de fuentes. También las cifras del distribuidor difieren en los distintos accesos. Esta situación obedece a que el mismo título está presente en distintas categorías temáticas y en diferentes subcategorías dentro de la misma categoría. Únicamente los listados alfabéticos por tipos de productos o el que se oferta a los intermediarios ofrecen datos de confianza. Conviene considerar, igualmente, la inestabilidad de los contenidos digitales, siendo el período de comienzo de año muy proclive a las nuevas incorporaciones, como hemos podido constatar.

En el caso de las revistas electrónicas aparecen contabilizadas las cerradas y aquéllas cerradas de las que el proveedor cuenta con el correspondiente backfile (destacamos 13 publicaciones con archivos perpetuos anteriores al año 1996). Incluso aparecen títulos cuyo contenido no está en línea, en cuyo caso la cifra asciende a 20 revistas.

Sorprende, como hemos adelantado, y en contraste con lo que sucede con otros distribuidores de contenidos, la alta densidad de libros electrónicos que puede responder a la política de difusión de la propia editorial que, disponiendo de los pre-prints y de la tecnología precisa, aprovecha las ventajas del formato electrónico en la distribución de contenidos.

En el caso de los libros electrónicos, la editorial recoge los diferentes volúmenes de una misma obra como títulos distintos, e incluso ediciones o reimpresiones di-

\begin{tabular}{|l|c|c|c|c|c|c|c||}
\hline & J & BF/C & B & RW & CP & DB & TOTAL \\
\hline Business & 43 & 6 & & 1 & & & 50 \\
\hline Chemistry & 63 & 14 & 117 & 12 & 3 & 4 & 213 \\
\hline Computer science & 10 & 6 & & 1 & & & 17 \\
\hline Earth and environmental science & 28 & & & & & & 28 \\
\hline Education & 11 & & & & & & 11 \\
\hline Engineering & 34 & 7 & 194 & 5 & & & 240 \\
\hline Law & 1 & 2 & & & & & 3 \\
\hline Life sciences & 62 & 4 & 49 & 5 & 8 & & 128 \\
\hline Mathematicals and statistics & 19 & 1 & 29 & & & & 49 \\
\hline Medicine & 61 & 10 & 78 & 3 & 2 & 1 & 155 \\
\hline Physics and astronomy & 11 & & 1 & 1 & & & 13 \\
\hline Polymers and materials science & 21 & 10 & 51 & 3 & & 1 & 86 \\
\hline Psycology & 25 & 4 & & 2 & & & 31 \\
\hline Social sciences & 1 & & & & & & 1 \\
\hline Total & \multicolumn{1}{|c|}{$390 \mathrm{~b}$} & 64 & 519 & 33 & 13 & 6 & 1.025 \\
\hline
\end{tabular}

versas se contabilizan de modo independiente. Parecería más conveniente, en un servicio de libros electrónicos, la actualización de contenidos eliminando versiones atrasadas.

La editorial añade dos bloques complementarios a las monografías y publicaciones periódicas que agrupa bajo los epígrafes Reference works (obras de referencia, enciclopedias, diccionarios, etc.) y Current protocols (manuales de laboratorio). Por último, Wiley distribuye, asimismo, bases de datos.

Advertidas estas consideraciones el cómputo total es el siguiente (figura 1):

—Revistas electrónicas (J): 390

-Backfiles y cerradas (BF/C): 64

—Libros electrónicos (B): 519

-Reference work (RW): 33

-Current protocols (CP):13

—Bases de datos (DB): 6

\section{-Items totales: 1025}

\section{Cobertura temática.}

Se analizan los contenidos presentes en las 14 categorías distinguiendo por tipo de fuente de información: Revistas abiertas, Backfiles y revistas cerradas, Reference works, Current protocols y Bases de datos. Wiley establece 14 categorías temáticas que agrupan contenidos correspondientes a las ciencias sociales, puras, ciencias aplicadas y de la naturaleza y de la salud, no recogiendo contenidos de humanidades (tabla 1).

Analizando los totales de la columna de la derecha se deduce que cuatro categorías: chemistry, engineering, life sciences y medicine representan el $71,80 \%$ de los contenidos totales. Cabe destacar el gran volumen de libros electrónicos en la categoría de ingeniería, siendo muy considerable el que se ofrece en química y medicina. Con respecto a los títulos de revista destacan química, ciencias de la vida y 
medicina con más de 60 títulos abiertos.

Resulta reseñable la existencia de dos categorías notablemente compensadas en cuanto a la distribución de tipos de materiales establecidos. Es el caso de química, donde cabe destacar el alto número de obras de referencia, más de un tercio del total, acaparando, igualmente, 4 de las 6 bases de datos. En cuanto a ciencias de la vida la inclusión de 8 current protocols, se corresponde con el $61,53 \%$ del total.

Podemos hablar de tres categorías con un volumen de contenidos intermedio: business, mathematical and statistics y polymers and material sciences. Dada la especificidad de esta última consideramos de mayor trascendencia los ítems que contiene, en particular el número de libros electrónicos. En cuanto a business destaca el alto número de publicaciones seriadas, resultando más equilibrada la categoría de mathematical and statistics.

Con respecto a las más pequeñas, cabe señalar el número aceptable de publicaciones periódicas de earth and environmental sciences con 28 , que representan el total de ítems de esta clase. Igualmente resulta correcto el número de seriadas dentro de psycology.

Son ciertamente pobres los contenidos de las cinco categorías restantes: computer science, education, law, physics and astronomy y social science que representan en su conjunto un $4,39 \%$ del total.

Parecería más adecuada la agrupación de categorías y el uso de etiquetas más compensadas, dado que resultan algunas muy genéricas y otras muy específicas. Cabe resaltar la inconsistencia de la categoría de ciencias sociales con un único ítem bajo un membrete muy extenso semánticamente. En esta clase podrían haberse agrupado los tres ítems de law, los 11 de education e incluso los 50 de business.

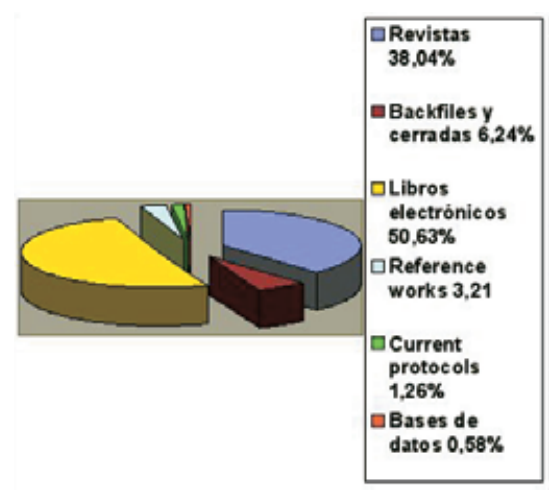

Figura 1

Esta situación se acentúa cuando se emplea el acceso general temático a los contenidos en los que aparecen subclases vacías, tal es el caso de marketing and sales, dentro de business, information and library science y special education dentro de education. Otros casos, con múltiples ejemplos, lo conforman subcategorías que ofrecen enlaces a títulos de otras subcategorías actuando no como clases temáticas sino como anclas o puntos de acceso: tres subclases de las ocho de business pueden ilustrar esta circunstancia, en esta categoría los títulos se concentran en una subcategoría denominada business ( $g$ eneral) denominación de la misma amplitud semántica que su clase principal. En el caso de computer sciences la subcategoría information technologies actúa curiosamente sólo como ancla, perteneciendo sus cuatro revistas a la subcategoría computer science (gene$\mathrm{ral}$ ) donde han optado por incluir la única revista de documentación, Journal of the American Society for Information Science and Technology (Jasist).

La accesibilidad del mismo título desde distintas categorías es una opción para facilitar la búsqueda, pero WIS introduce además apartados de "Revistas relacionadas" que cumplen idéntica función, establecer vínculos, por lo que sería deseable un mayor grado de consistencia que favorezca la ex- ploración temática sin dispersar contenidos.

Si analizamos la tabla 1 por tipos de fuentes de información apreciamos que todas las categorías incluyen publicaciones periódicas y, sin embargo, solamente aparecen obras monográficas en los apartados de ciencias puras, aplicadas y ciencias de la naturaleza y de la salud.

\section{Cobertura vertical.}

La fecha clave en la incorporación de las revistas se sitúa en el año 1996 al que corresponden como fecha de inicio un $55,89 \%$ del total de las publicaciones abiertas ofrecidas. Anecdótica resulta la incorporación de una revista desde 1991 correspondiente a la categoría de medicina: Ultrasound in obstetrics and gynecology.

Resulta reseñable la incorporación de contenidos de 5 nuevas revistas en los meses de enero/febrero de 2004 fecha en la que se ha realizado el estudio empírico del proveedor. WIS anuncia que son 17 las incorporaciones previstas para el presente año.

Podemos establecer tres periodos temporales atendiendo a la fecha a partir de la cual se encuentran disponibles los contenidos de los títulos de revistas suministrados en este paquete, tal como queda reflejado en el gráfico de la figura 2.

Conviene subrayar la disposición de los backfiles de 46 títulos, así como de archivos perpetuos anteriores al año 1996 en el caso de 13 títulos de revistas, 8 de los cuales corresponden a la categoría de

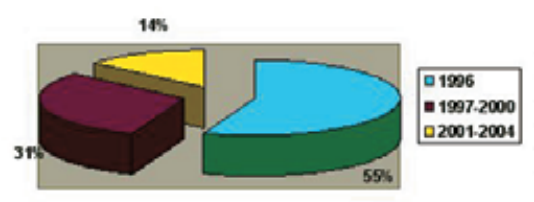

Figura 2 
polímeros donde existen contenidos desde la década de los 40 del pasado siglo.

Los libros electrónicos distribuidos han sido publicados con posterioridad a 1991, el número de títulos editados hasta el año 1999 representa un escaso $10 \%$ del total. El repunte comienza en 2000 y se produce una clara concentración de obras cuyo copyright se sitúa en 2001 y 2002, con un porcentaje también elevado en 2003. El vértice recae en 2002 con 198 títulos, lo que supone más de un $38 \%$ del total.

\section{Arquitectura de acceso}

\section{Sistemas de control de ac- ceso empleado.}

Wiley emplea la autenticación de direcciones IP y cuenta con los denominados Multi-site global coverage, acceso previsto desde diversos campus de la misma institución, y el Roaming Access para permitir a los investigadores el acceso fuera de su institución, previsto únicamente en el caso de licencias de acceso mejorado (EAL). Hace uso de cookies temporales de control de sesión y, de modo opcional, de otras permanentes en el caso de usuarios registrados.

\section{Prestaciones de búsqueda.}

Se establece una clara distinción entre la búsqueda navegacional (browsing) y la recuperación empleando un motor de búsqueda (searching), que actúa sobre todos los contenidos distribuidos por la editorial.

La búsqueda navegacional parte de dos opciones: la primera, resaltada claramente por la empresa, pretende conducir al usuario a los contenidos, sin distinción inicial de productos empleando una serie de categorías y subcategorías temáticas que llevan al título de la revista, libro electrónico, obra de referencia, base de datos o manual de laboratorio. La otra alternativa per-

mite la exploración partiendo de la selección previa del tipo de producto, agrupando de nuevo temáticamente los contenidos en categorías y subcategorías y añadiendo a los productos ya citados el apartado de "Colecciones".

La herramienta de recuperación de esta editorial cuenta con un formulario de consulta sencilla, disponible directamente en la página de entrada y otro para la avanzada. La caja de búsqueda simple permite explicitar el/los término/s y realizar la búsqueda sobre el conjunto de los contenidos o solamente sobre los títulos específicos de los mismos.

El formulario de búsqueda avanzada cuenta con posibilidades de selección de campos (autor, afiliación del autor, título de la publicación, título del artículo, DOI, ISSN, etc.) permitiendo, igualmente, delimitar por tipo de producto, por colección e ítems salvados, por materias que se corresponden con las categorías temáticas y por rango de fechas.

\section{«En el caso de los li- bros electrónicos, la editorial recoge los diferentes volúmenes de una misma obra como títulos distin- tos, e incluso edicio- nes o reimpresiones diversas se contabili- zan de modo inde- pendiente»}

Dispone de operadores booleanos, de proximidad, distancia y truncamiento, es posible realizar búsquedas por frase y emplear paréntesis. No distingue entre mayúsculas y minúsculas e incrementa automáticamente la búsqueda haciendo uso de técnicas de stemming.

Existen, además, prestaciones de búsqueda de notable interés que complementan, sin duda, las ya señaladas. Así, desde las páginas de resultados se accede a la búsqueda por citas (sobre la que incidiremos más adelante), a la limitación de la consulta por medio de una caja de búsqueda prevista para acotar términos dentro de la correspondiente publicación y a la búsqueda de artículos relacionados para lo que la editorial tiene en cuenta tanto la autoría como la temática.

La mayor parte de las obras de referencia, bases de datos y productos especializados incluyen, además de la búsqueda estándar, otra avanzada dentro del producto concreto que permite realizar consultas más específicas y que se adaptan a los contenidos particulares del mismo.

Conviene reseñar, asimismo, los interesantes enlaces navegacionales que se adaptan a la casuística de los tipos de productos. Así, en el caso de las obras de referencia, el sistema nos permite navegar por materia y por el sumario de los contenidos. En las bases de datos es posible explorar por contenidos, artículos, títulos, materias, etc.

Por último, es posible reutilizar las estrategias de búsqueda (edit search) o guardarlas, aunque sólo se encuentra activa esta herramienta para los usuarios previamente registrados.

\section{Presentación de resulta- dos.}

Permite la ordenación de resultados por relevancia - con valores entre .01 (partial match) y 1.00 (exact match)- por fechas o por título de la publicación. Muestra por defecto, si no se ha señalado un tipo de producto concreto, los resultados de artículos de revistas, presentando un menú superior para que el usuario pueda lanzar la misma búsqueda en el resto de fuentes de información. 
En la presentación abreviada aparece el listado de resultados con los campos de descripción formal fundamentales: título, datos de la publicación y autor, así como el enlace al abstract, a las referencias $y$ al texto completo correspondiente, en el que se precisa su extensión en $\mathrm{Kb}$.

El formato preferido para la presentación del documento final mayoritariamente es el pdf en un $97,30 \%$ complementado en 78 publicaciones con la presentación en html, lo que representa un $17,41 \%$.

\section{Características de la interfaz}

\section{Diseño.}

Podemos hablar de una correcta legibilidad y visualización de los contenidos. La página de entrada denota preferencia por la navegación temática frente a aquella basada en tipos de productos, desplegada en la zona inferior y sólo visible haciendo uso del scroll.

Se observa, igualmente, una inclinación hacia el uso de formularios, botones de radio, cajas de validación o listas de opciones frente al uso directo de operadores de rango o booleanos; esta facilidad de utilización se ve favorecida por los desplegables con estos operadores entre cajas de búsqueda.

Valoramos positivamente el resalte de los términos de búsqueda especificando el/los campo/s de búsqueda y el tipo de producto en su caso.

\section{Ergonomía.}

Para la selección de ítems en la lista de resultados se emplean sencillas e intuitivas cajas de validación complementadas con la posibilidad de pasar a visualizar los documentos seleccionados o sus resúmenes.

La carga de las páginas se efectúa con rapidez. Las soluciones de descarga e impresión de registros

vienen determinadas por el formato final del documento. Cuando éste aparece en html se acompaña de iconos que conducen a su impresión o envío por correo electrónico.

\section{«Se establece una clara distinción entre la búsqueda navega- cional y la recupera- ción empleando un motor de búsqueda que actúa sobre to- dos los contenidos distribuidos por la editorial»}

En cuanto a aspectos relacionados con la personalización de la interfaz, dependen en gran medida de la creación de perfiles de usuario. No se contempla, en cualquier caso, la posibilidad de configurarla en castellano.

\section{Amigabilidad.}

Proporciona información sobre la compañía, presenta una ayuda general adecuada sobre el uso de WIS, ayuda específica sobre las opciones de búsqueda complementada con ayudas contextualizadas y la posibilidad de acceder a una versión de demostración.

Creemos destacable el hecho de insertar la caja de búsqueda sencilla como elemento común que aparece en la zona superior derecha de cualquier página del sitio, evitando que el usuario haya de regresar a la página de búsqueda inicial.

Supone una ayuda inestimable para el usuario la organización navegacional temática en categorías y subcategorías así como la integración de numerosos elementos hipertextuales dispuestos para incrementar o delimitar búsquedas previas. Consideramos interesantes los apartados dedicados a "Productos relacionados" a los que se accede desde las subcategorías temáticas y desde los accesos diferenciados por productos. Sin em- bargo, como ya hemos advertido específicamente al tratar la cobertura horizontal, sería necesaria una unificación de criterios en el establecimiento de relaciones.

\section{Funcionalidades y servicios de valor añadido}

A las prestaciones ya tradicionales de creación de perfiles de usuario, servicio de alertas o DSI, previstas para usuarios registrados, se suman otras como la creación de paquetes de recursos pedagógicos seleccionados, funcionalidades como EarlyView que ofrece preprints de 192 publicaciones o las colecciones de backfile retrospectivas en cuyo desarrollo ha incidido la empresa desde el pasado 2003.

También interesantes son las utilidades de recuperación a demanda de documentos no suscritos, artículos o capítulos de libros; se proporciona así una mayor flexibilidad en las modalidades de acceso a los contenidos tratando de adaptarse a necesidades de usuarios diversos, son los pay-per-view y el servicio ArticleSelect mediante el cual se ofrecen bonos o vales con características diferentes ya se trate de una suscripción $E A L$ o $B A L$. En el primer caso, cuando un usuario gasta en vales el $115 \%$ del precio de suscripción de la revista, Wiley le considera suscrito a ella durante ese año.

Destacan también las secciones "Top de artículos", "Nuevos títulos incorporados" (a medio camino entre la estrategia comercial y la difusión de la información) o el ya mencionado de "Revistas relacionadas" que sirve para trazar vínculos entre contenidos.

Mención especial merece el apartado concreto de "Productos y servicios para bibliotecarios" donde se aclaran aspectos fundamentales para la adecuada gestión de la colección: opciones de licencias 
previstas, de métodos de autenticación de usuarios, realización de estadísticas, integración de obras en los opacs, etc.

Reseñamos la sección que ofrece la editorial con información dirigida a los autores sobre las pautas para la publicación de trabajos $\mathrm{y}$, por último, el servicio denominado MobileEdition mediante el cual distribuye tablas de contenidos y abstracts directamente a dispositivos PDA (Personal Digital Assistant) empleando tecnología Palm.

El sistema de citas reviste solidez. La editorial emplea como identificador persistente el modelo DOI (Digital Object Identifier) que en la descripción aparece como campo recuperable. Wiley hace uso del software Crossref como resolutor para asociar identificadores con direcciones, utilizándose en 357 publicaciones $(79,68 \%)$.

El tratamiento documental de la empresa faculta el uso de funcionalidades bibliométricas derivadas de las búsquedas de referencias $\mathrm{y}$ citas.

Con respecto a los derechos de archivo, una vez concluido el período de validez de la licencia, Wiley facilitará el acceso al tex to completo de las publicaciones electrónicas cubiertas y publicadas durante su plazo de validez, ya sea mediante la prolongación del acceso en línea a dicho material en el servidor de WIS o a través de una copia archivada en un medio electrónico seleccionado por Wiley, por una cuota basada en el coste del servicio y que deberán acordar las partes.

\section{Conclusiones}

Se perciben ciertas lagunas informativas en la licencia suscrita, de crucial relevancia en algunos casos como la especificación de títulos de revistas contratados y características detalladas de los mis- mos, que debieran figurar en los anexos.

Los contenidos proporcionados por WIS se distribuyen en las siguientes categorías temáticas: ciencias sociales $(9,36 \%)$, ciencias puras $(25,56 \%)$, ciencias de la naturaleza y de la salud $(31,60 \%)$ y ciencias aplicadas $(33,95 \%)$. Para la consecución de estos porcentajes hemos agrupado las clases establecidas por la empresa de la siguiente forma:

-Ciencias sociales: business, education, law, psycology y social sciences.

-Ciencias puras: chemistry, mathematicals and statistics y physics and astronomy.

- Ciencias de la naturaleza y la salud: earth and environmental science, life sciences y medicine.

-Ciencias aplicadas: computer science, engineering y polymers and materials science.

Se trata, por tanto, de un distribuidor de contenidos científicotécnicos fundamentalmente, con una escasa cobertura en el apartado de ciencias sociales.

Valoramos positivamente el reconocimiento de IPs frente al uso de contraseñas de usuario, la rápida actualización de contenidos, el mantenimiento de los mismos, su exhaustividad, el crecimiento de backfiles o el lanzamiento de colecciones temáticas. No podemos obviar, no obstante, la limitación que supone el sesgo idiomático.

Las características actuales de las monografías digitalizadas hacen pensar más en un complemento a la labor editorial de la propia empresa disponiendo los textos en formato pdf que en una apuesta por la edición de e-books explotando las particularidades que estos productos han de implementar. En el caso de las revistas, sólo un $17 \%$ de las mismas introducen los contenidos en formato html facilitando funcionalidades de enlazado que en pdf no son factibles.

La interfaz presentada por WIS resulta adecuada en los tres parámetros considerados de diseño, ergonomía y amigabilidad. Los servicios de valor añadido se hallan ligados, en gran medida, a los registros de usuario.

Conviene señalar que no se han abordado determinados aspectos evaluativos, ya sea por requerir de un estudio comparativo entre proveedores o bien por necesitar un marco de referencia, biblioteca o consorcio. Es preciso establecer el grado de pertinencia de los paquetes en relación con las áreas de conocimiento de las universidades y de las titulaciones que imparten así como el grado de solapamiento entre unos y otros.

Resulta imprescindible abordar el estudio de costes en relación a la cobertura proporcionada, a la calidad de las publicaciones suministradas, a la sencillez de uso de la interfaz y valores añadidos proporcionados así como las relaciones entre uso y coste (gasto por consulta, descargas, etc.). Sólo datos estadísticos normalizados, como los ofrecidos por el modelo del proyecto Counter (Counting Online Usage of NeTworked Electronic Resources) permitirán evaluaciones cuantitativas rigurosas.

Las relaciones entre recursos, oferta de contenidos y uso han de ser también consideradas. Es necesario asegurar que más recursos (más inversión) se traduzcan en mayor oferta de contenidos y ésta en un uso creciente. Una evaluación completa habría de establecer indicadores de eficiencia (oferta/recursos), adecuación (demanda/oferta) y transmisión (recursos/demanda).

No podemos olvidar tampoco la relación existente entre el uso, las facilidades de la interfaz y la formación de usuarios. Por último, resul- 


\section{(0) baratz}

En primera línea en

Sistemas de Información y Gestión del Conocimiento
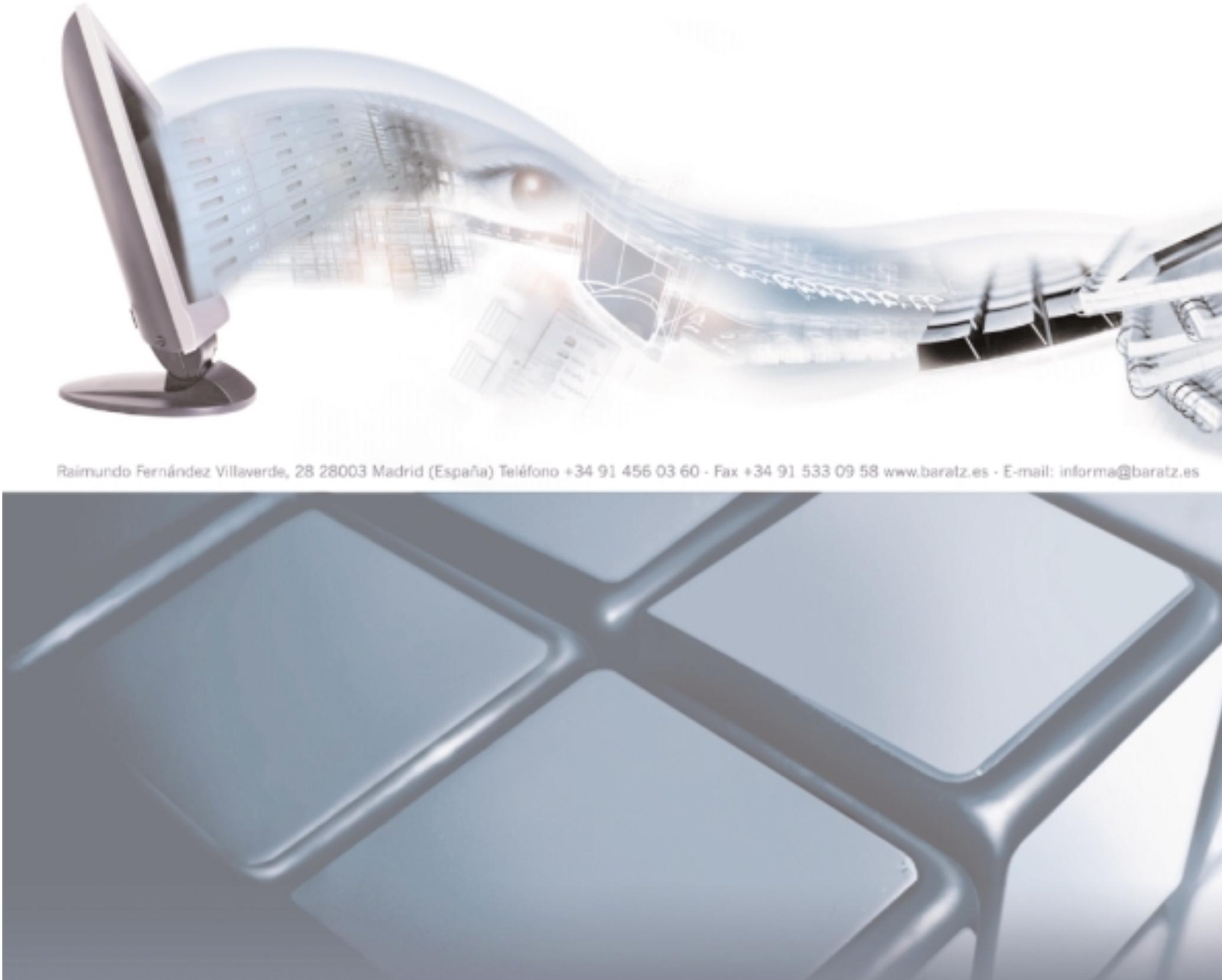

- Soluciones para bibliotecas: Absys, absys NET, Absys

- Gestión documental y del conocimiento: BKM, Baratz Windows, BRSCGI

- Soluciones para archivos: Albalá

- Servicios de Catalogación Retrospectiva

- Edición en CD-Rom

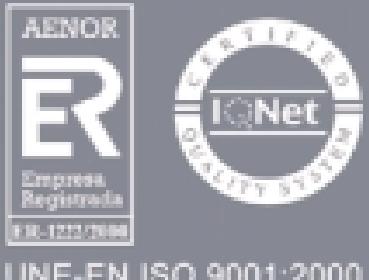


taría conveniente conocer el impacto que la utilización de los contenidos electrónicos revierte en la investigación de las universidades.

Solamente procesos evaluativos de este tipo permitirán la negociación con las grandes multinacionales del negocio editorial fundamentada en criterios objetivos de calidad y uso protegiendo la infraestructura de provisión de contenidos del país y permitiendo a las universidades y a sus bibliotecas el mantenimiento de su liderazgo en la provisión de contenidos científicos. Sugerimos, por tanto, evaluar para evolucionar en la configuración de una adecuada infraestructura que garantice el acceso a la información científico-técnica.

\section{Nota}

1. Evaluación de los proveedores de revistas electrónicas y estudio de la calidad de las publicaciones digitales (2004-2005). Investigador principal: Dra. Rodríguez Bravo.

\section{Bibliografía}

Acebes, R.; Palafox Parejo, M.; Tardón, E. Curso de acceso y gestión de los recursos electrónicos en las bibliotecas: León, 24 a 26 de febrero de 2004, Sedic, 2004.

Cantos Puig, C.; Casaldáliga Riera, A.; Fernando Quer, N. "Las revistas electrónicas en la biblioteca de la Universitat Pompeu Fabra: acceso, gestión y difusión”. En: $3^{a}$ Jornadas andaluzas de documentación: Jadoc'03. Sevi1la: Asociación Andaluza de Documentalistas, 2003, pp. 59-68.

Codina, L. "Evaluación de recursos digitales en línea: conceptos, indicadores y métodos". En: Revista española de documentación científica, 2000, v. 23, n. 1, pp. 9-44.
Counter (Counting Online Usage of Networked Electronic Resources). Consultado en: 09-0204.

http://www.projectcounter.org/

Jewell, T. D. Selection and presentation of commercially available electronic resources: issues and practices. Washington, D. C.: Digital Library Federation, 2001. Consultado en: 12-01-04.

http://www.clir.org/pubs/reports/pub99/pub99.pdf

Martín González, J. C.; Merlo Vega, J. A. "Proveedores comerciales de revistas electrónicas de ciencias sociales". En: El profesional de la información, 2003, v. 12, n. 5, pp. 415-419.

Rodríguez Bravo, B. ; Alvite Díez, M. L. Propuesta metodológica de evaluación de interfaces de OPACs. INNOPAC versus UNICORN. Revista Española de Documentación Científica, 2004, vol.27, n. 1, enero-marzo, pp. 30-44.

$M^{a}$ Luisa Alvite Díez; Blanca Rodríguez Bravo, Área de Biblioteconomía y Documentación, Universidad de León. luisa.alvite@unileon.es dphbrb@unileon.es 